\title{
A NOTE ON UNIQUENESS AND CONVERGENCE OF SUCCESSIVE APPROXIMATIONS
}

\author{
Fred Brauer
}

(received April 8, 1958)

1. In a recent note in this Bulletin [3], W.A.J. Luxemburg has shown in two different ways that a condition of Krein and Krasnosel'skii [2] for the uniqueness of solutions of a differential equation also implies the convergence of the successive approximations. Here, a third proof of the uniqueness and the convergence of successive approximations, formulated for systems of differential equations, will be obtained. This third proof is modelled on the methods used in proving general uniqueness and convergence theorems [1]. The approach is suggested by Luxemburg's idea of breaking the argument into two stages and using one of the hypotheses in each stage. Since the proofs given here are hardly shorter than the earlier direct proofs, their main interest lies in the fact that they fit what appeared to be an isolated result into the framework of a general theory.

2. We consider the initial value problem

$$
x^{\prime}=f(t, x), \quad x(0)=0,
$$

where $x$ and $f$ are $n$-dimensional vectors. The norm $|x|$ of any vector $x$ is defined in the usual way (cf. [1]) as the sum of the absolute values of its components. We consider a vector function $f(t, x)$ satisfying the pair of conditions

$$
\begin{aligned}
& \left|f\left(t, x_{1}\right)-f\left(t, x_{2}\right)\right| \leqq A\left|x_{1}-x_{2}\right|^{\alpha}, \\
& \left|f\left(t, x_{1}\right)-f\left(t, x_{2}\right)\right| \leqq k\left|x_{1}-x_{2}\right| / t,
\end{aligned}
$$

where $k, A$, and $\alpha$ are constants with $0<k, 0<\alpha<1$.

THEOREM $1[2,3]$. Let $f(t, x)$ be continuous in a region $0 \leqq t<a,|x|<b$, and bounded in norm by $M$ in this region.

Can. Math. Bull., vol.2, no. 1, Jan. 1959 
Suppose also that $f$ satisfies (2) and (3) in this region, with $k(1-\alpha)<1$. Then there is a unique solution of $(1)$ on $0 \leqq t<a$.

Proof. Suppose there are two solutions $x_{1}(t)$ and $x_{2}(t)$ of $(1)$ on $0 \leqq t<a$, and let $m(t)=\left|x_{1}(t)-x_{2}(t)\right|$. We must show that $m(t)=0$ for $0 \leqq t<a$. We have

$$
\begin{aligned}
\left|m^{\prime}(t)\right| & \leqq\left|x_{1}{ }^{\prime}(t)-x_{2}{ }^{\prime}(t)\right| \leqq\left|f\left(t, x_{1}(t)\right)-f\left(t, x_{2}(t)\right)\right| \\
& \leqq A\left|x_{1}(t)-x_{2}(t)\right|^{\alpha} \leqq A[m(t)]^{\alpha},
\end{aligned}
$$

using (2). Suppose there exists $\sigma, 0<\sigma<a$, such that

$$
m(\sigma)>[A(1-\alpha) \sigma]^{1 /(1-\alpha)} .
$$

Then there is a solution $u(t)$ of the differential equation

$$
\mathrm{u}^{\prime}=\mathrm{Au}^{\alpha},
$$

passing through the point $(\sigma, \mathrm{m}(\sigma))$, and existing on some interval to the left of $\sigma$. As far to the left of $\sigma$ as $u(t)$ exists,

$$
u(t) \leqq m(t) .
$$

If this is false, there exists $\xi \leqslant \sigma$ such that $u(\xi)=m(\xi)$ and $u(t)>m(t)$ for $t<\xi$ and $t$ sufficiently near $\xi$, say for $\xi-h \leqq t<\xi$. But if $\xi-h \leqq t<\xi$, then $u^{\prime}(t)=A[u(t)]^{\alpha}>A[m(t)]^{\alpha} \approx m^{\prime}(t)$. This, together with $m(\xi-h)<u(\xi-h)$ implies $m(\xi)<u(\xi)$, a contradiction which proves (7). The solution $u(t)$ can be continued to $t=0$. If $u(c)=0$, $0<c<\sigma$, we can effect the continuation by defining $u(t)=0$ for $0<\mathrm{t}<\mathrm{c}$; otherwise (7) ensures that the continuation is possible. Since $m(0)=0, \lim _{t \rightarrow 0} u(t)=0$, and we define $u(0)=0$. Now we have a solution $u(t)$ of $(6)$ on $0 \leq t \leq \sigma$ satisfying $u(0)=0$. But $(6)$ can be solved explicitly, and any solution with $u(0)=0$ has the

form $u(t)=0,[0 \leqq t \leqq c], u(t)=[A(1-\alpha)(t-c)]^{1 /(1-\alpha)},[t>c]$, and satisfies

$$
u(t)=[A(1-\alpha) t]^{1 /(1-\alpha)}, \quad 0 \leqq t \leqq \sigma .
$$

Then $u(\sigma)=m(\sigma),(5)$ and (8) yield a contradiction, and (5) can not be true for any $\sigma$. Therefore

$$
m(t) \leqq[A(1-\alpha) t]^{1 /(1-\alpha)}, \quad 0 \leqq t<a,
$$


and since $k(1-\alpha)<1$, we have

$$
\lim _{t \rightarrow 0^{t}} t^{-k} m(t)=0 \text {. }
$$

To complete the proof, we must show that (10) implies $m(t) \equiv 0$. Proceeding as in the first part of the proof, but using (3) instead of (2), we obtain $\left|\mathrm{m}^{\prime}(\mathrm{t})\right| \leq \mathrm{km}(\mathrm{t}) / \mathrm{t}$. Suppose $\mathrm{m}(\tau) \neq 0$ for some $\tau$, $0<\tau<a$. The same argument as that used above, replacing (6) by

$$
\mathrm{v}^{\prime}=\mathrm{kv} / \mathrm{t},
$$

gives a solution $v(t)$ of (11) on $0 \leqq t \leqq \tau$ such that $v(\tau)=m(\tau), 0 \leqq v(t)$ $\leqq m(t), v(0)=0$. Then $\lim _{t \rightarrow 0} t^{-k} v(t) \leqq \lim _{t \rightarrow 0^{t}} t^{-k} m(t)=0$, and

$$
\lim _{t \rightarrow 0^{t}}-k v(t)=0 \text {. }
$$

But the only solution $v(t)$ of (11) satisfying (12) is the identically zero solution. This contradicts $\mathrm{v}(\tau)=\mathrm{m}(\tau) \neq 0$, and thus $\mathrm{m}(\mathrm{t})=0$, $0 \leqq t<a$, which completes the proof of the theorem.

3. The successive approximations to the solution of (1) are defined by

$$
x_{0}(t)=0, x_{j+1}(t)=\int_{0}^{t} f\left(s, x_{j}(s)\right) d s, \quad(j=0,1, \ldots)
$$

THEOREM 2 [3]. Let $f$ satisfy the hypotheses of Theorem 1. Then the successive approximations (13) converge uniformly on $0 \leqq t<\min (a, b / M)$ to the unique solution of $(1)$.

Proof. An examination of the proof of the general theorem on the convergence of successive approximations ( $[1]$, chap. 2 , Theorem 3.1) shows that the approximations (13) form a uniformly bounded equicontinuous sequence, and that the conclusion of the theorem is equivalent to

$$
\lim \sup _{j \rightarrow \infty}\left|x_{j+1}(t)-x_{j}(t)\right|=0, \quad 0 \leqq t<\min (a, b / M) .
$$

Let $w_{j}(t)=x_{j+1}(t)-x_{j}(t), m(t)=\lim \sup _{j \rightarrow \infty}\left|w_{j}(t)\right|$. Then m $(0)=0$, and $m(t)$ is continuous on $0 \leqq t<\min (a, b / M)$, since it is the upper limit of a uniformly bounded equicontinuous sequence of functions. Using (13) and (2), we obtain

$$
\begin{aligned}
\left|w_{j+1}(t+h)-w_{j+1}(t)\right| \leqq \int_{t}^{t+h}\left|f\left(s, x_{j+1}(s)\right)-f\left(s, x_{j}(s)\right)\right| d s & \\
& \leqq \int_{t}^{t+h} A\left|w_{j}(s)\right|^{\alpha} d s .
\end{aligned}
$$


Because of the continuity of $m$ and the equicontinuity of $w_{j}$, given any $\varepsilon>0$ there exists an integer $M(\varepsilon)$, independent of $s$ and $\mathrm{j}$ such that

$$
\left|w_{j}(s)\right| \leqq m(s)+\varepsilon, \quad j>M(\varepsilon) \text {. }
$$

It follows from (15) and (16) that

$$
\left|w_{j+1}(t+h)-w_{j+1}(t)\right| \leqq \int_{t}^{t+h} A|m(s)+\varepsilon|^{\alpha} d s, \quad j>M(\varepsilon)
$$

From the definition of $m$ it is easy to see that $|m(t+h)-m(t)|$ $\leqq \lim \sup _{j \rightarrow \infty}\left|w_{j+1}(t+h)-w_{j+1}(t)\right| \cdot$ Combining this with (17) and then letting $\varepsilon \rightarrow 0$, we obtain

$$
|m(t+h)-m(t)| \leqq \int_{t}{ }^{t+h} A|m(s)|^{\alpha} d s .
$$

The inequality (18) implies that $m^{\prime}(t)$ exists on any interval $(t, t+h)$, $t \geqq 0$, and that $\left|\mathrm{m}^{\top}(t)\right| \leqq A|\mathrm{~m}(t)|^{\alpha}$. The argument used in the proof of Theorem 1 beginning with (4) proves

$$
\lim _{t \rightarrow 0^{t-k}} \operatorname{m}(t)=0
$$

To complete the proof, we must show that (19) implies $m(t) \equiv 0$. This is done in much the same way as the last stage of the proof of Theorem 1. Suppose $m(\tau) \neq 0$ for some $\tau, 0<\tau<a$. A repetition of the first part of the proof, using (3) instead of (2), so that (11) replaces (6), gives a solution $v(t)$ of $(11)$ on $0 \leqq t \leqq \tau$ such that $\mathrm{v}(\tau)=\mathrm{m}(\tau), 0 \leqq \mathrm{v}(\mathrm{t}) \leqq \mathrm{m}(\mathrm{t}), \mathrm{v}(0)=0$. Then (19) implies (12), and the desired conclusion follows as in Theorem 1 .

\section{REFERENCES}

1. E.A. Coddington and Norman Levinson, Theory of ordinary differential equations, (New York, 1955).

2. M.A. Krasnosel'ski theorems for the equation $y^{\prime}=f(x, y)$, Uspehi Mat. Nauk. (N.S.) 11 (1956), 209-213 (Russian).

3. W.A.J. Luxemburg, On the convergence of successive approximations in the theory of ordinary differential equations, Canadian Math. Bulletin 1 (1958), 9-20.

University of Chicago and University of British Columbia 